\title{
Optical waveform tailoring in passive and laser cavity fibre systems
}

\author{
Sonia Boscolo ${ }^{1}$, Ilya Gukov ${ }^{2}$, and Christophe Finot ${ }^{3}$ \\ ${ }^{1}$ Aston Institute of Photonic Technologies, School of Engineering and Applied Science, Aston University, \\ Birmingham B4 7ET, United Kingdom \\ ${ }^{2}$ Moscow Institute of Physics and Technology \& Skolkovo Institute of Science and Technology, Moscow, Russia \\ ${ }^{3}$ Laboratoire Interdisciplinaire Carnot de Bourgogne, UMR 6303 CNRS - Université Bourgogne Franche- \\ Comté, Dijon, France \\ christophe.finot@u-bourgogne.fr
}

\begin{abstract}
The interplay among the effects of dispersion, nonlinearity and gain/loss in optical fibres is a powerful tool to generate a broad range of pulse shapes with tuneable properties. Here we propose a method to optimise the systems parameters for a given pulse target. By reducing the system complexity and applying machine-learning strategies, we show that it is possible to efficiently identify the sets of parameters of interest. Two configurations are numerically investigated: pulse shaping in a passive normally dispersive fibre and pulse generation in a dualpump nonlinear-amplifying-loop-mirror mode-locked fibre laser.
\end{abstract}

Keywords: pulse shaping; nonlinear fibre optics; optical processing; fibre lasers.

\section{INTRODUCTION}

In recent years, there has been a growing interest from the photonics community in the generation of nonconventional optical waveforms because of their applications in all-optical signal processing and microwave signal manipulation. While sinusoidal, Gaussian and hyperbolic secant intensity profiles are now routinely produced by modulators or mode-locked lasers, other signal waveforms such as parabolic, triangular or flat-top pulse shapes remain rather hard to synthesise. Several approaches to the generation of specialised waveforms have been explored, including linear spectral shaping, the use of special Mach-Zehnder modulator architectures [1], and coherent Fourier synthesis [2,3], each presenting its own advantages and drawbacks. The latter include the impossibility to increase the pulse bandwidth intrinsic to the linear approach, poor energy efficiency, and the need for a feedback loop.

The interplay among the effects of dispersion, nonlinearity and gain/loss in optical fibre systems can be efficiently used to shape the pulses and manipulate and control the light dynamics and, hence, lead to different pulse-shaping regimes. In particular, it has been demonstrated that the temporal and spectral properties of a pulse can be tailored by use of such a nonlinear shaping approach to generate ultra-short parabolic, rectangular or triangular pulses [4-7]. However, achieving a precise waveform with various prescribed characteristics, such as temporal duration and chirp properties, is a complex issue that requires careful choice of the initial pulse conditions and system parameters. The general problem of optimisation towards a target operational regime in a complex multi-parameter space can be intelligently addressed by implementing machine-learning strategies.

In this paper, we discuss a novel approach to the characterisation and optimisation of nonlinear shaping in fibre systems, which combines numerical simulations of the basic propagation model to identify the relevant parameters with the machine-learning method of neural networks (NNs) to make predictions across a larger range of the data domain.

\section{PULSE SCULPURING IN PASSIVE NORMALLY DISPERSIVE FIBRE}

Firstly, we tackle the general problem of determining the parameters of nonlinear pulse shaping systems based on pulse propagation in a passive normally dispersive fibre that are required to achieve the generation of pulses with different, simultaneously optimised temporal features. A scheme for nonlinear shaping typically comprises a pre-chirping stage, in which an initial pulse $\psi_{0}(t)$ with a peak power $P_{0}$ and a full-width at half maximum (fwhm) duration $T_{\text {in }}$ is propagated through a dispersive medium to acquire a parabolic spectral phase characterised by a chirp coefficient $C_{0}$, followed by nonlinear propagation of the chirped pulse through a normally dispersive fibre that reshapes both its temporal and spectral intensity profiles. Pulse propagation in the fibre system follows the standard nonlinear Schrödinger equation (NLSE) [8]:

$$
i \frac{\partial u}{\partial \xi}-\frac{1}{2} \frac{\partial^{2} u}{\partial \tau^{2}}+N^{2}|u|^{2} u=0
$$

Here, we use the dimensionless variables: $u=\psi / \sqrt{P_{0}}, \xi=z / L_{D}$, and $\tau=t / T_{0}$, where $\psi(z, t)$ is the complex envelope of the pulse, $z$ is the propagation coordinate, $t$ is the retarded time, $L_{D}=T_{0}{ }^{2} /\left|\beta_{2}\right|$ and $L_{N L}=1 /\left(\gamma P_{0}\right)$ are 
the respective dispersion length and nonlinear length associated with the pulse at the input to the system, the parameter $N$ ('soliton-order' number) is introduced as $N^{2}=L_{D} / L_{N L}$, and $\beta_{2}$ and $\gamma$ are the respective groupvelocity dispersion and Kerr nonlinearity parameter of the fibre. This way, the search for the best system parameters to achieve specified output pulse characteristics is trimmed to a three-dimensional optimisation problem in the space of $(C, N, \xi)$, where $C=C_{0} T_{0}$ is the normalised chirp coefficient of the pulse at the entrance of the fibre. Hence, the parameter search is apparently made significantly more efficient, and for a specific selected set $(C, N, \xi)$ there are many groups of practical parameters suitable the defining equations of $C, N$, and $\xi$. We use a graphical method to solve this optimisation problem, by which the intersections of characteristic surfaces in the space of $(C, N, \xi)$ obtained from direct numerical simulations provide the means to quickly identify the sets of parameters of interest. An example is provided in Fig. 1(a), which shows the different regions of parameter space supporting the formation of a parabolic pulse with a prescribed duration. The optimum region as pinpointed by the highest Strehl ratio values, is the one that features anomalous input chirping and rather high input powers, underpinning nonlinear pulse dynamics dominated by a spectral compression process. The results obtained with the NN training method are plotted in Fig. 1(b) and show good agreement with the NLSE simulation results. In this case, a regression model based on a NN algorithm is trained on a reduced-size set of data produced from direct numerical simulations to identify the target output pulse states across the full range of parameter variation [9]. Figure 2 shows examples of the generation of parabolic, triangular and nearly rectangular pulse waveforms of a certain desired duration. In all cases, the spectrum extent can also be controlled.

(a)

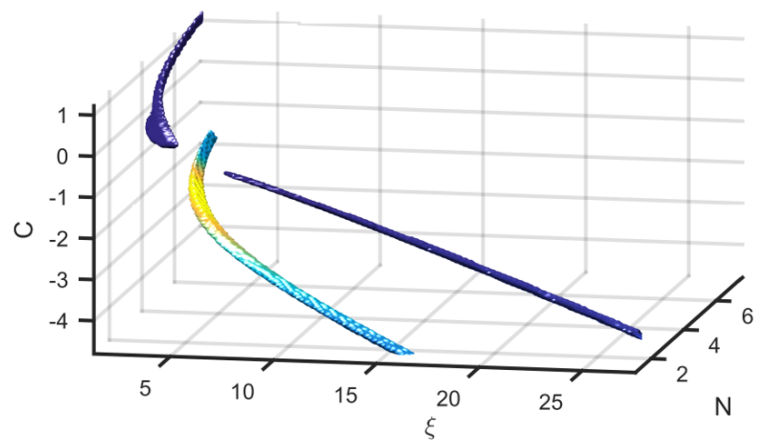

Strehl ratio

$.3 \quad .5 \quad .7$

(b)

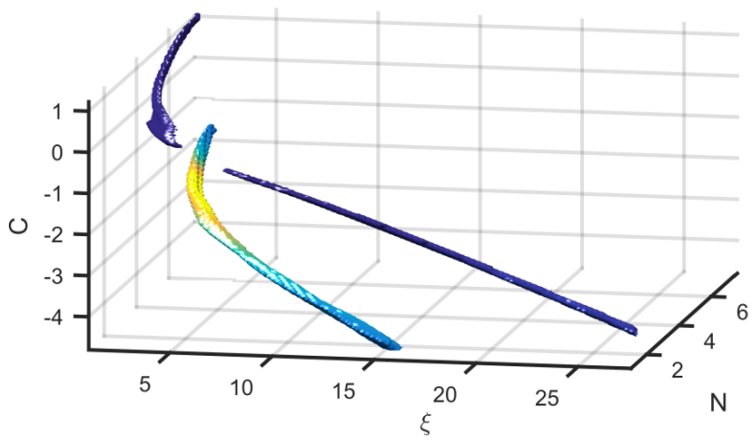

Figure 1. Pulse shaping in passive normally dispersive fibre. Crossings of parameter surfaces in the threedimensional parameter space supporting the formation of a parabolic waveform with duration of $10 T_{i n}$. The results obtained from numerical simulations of the NLSE propagation model [subplot (a)] are compared with the results generated by the $N N$ regression model [subplot (b)].

parabolic pulse

$C=-2, N=5, \breve{\xi}=3$
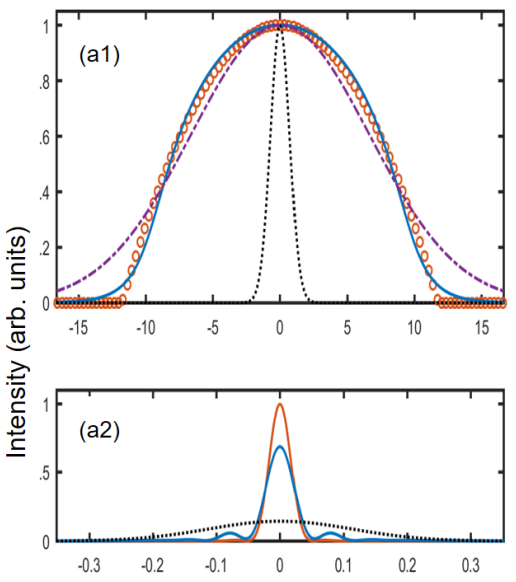

triangular pulse

$C=-1, N=7.25, \xi=11,5$
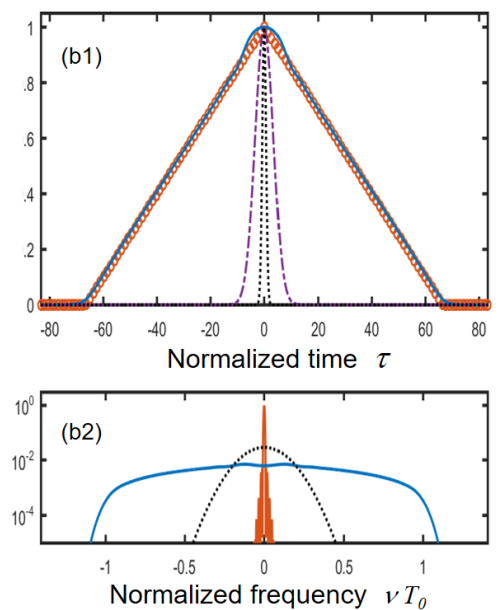

rectangular pulse

$C=-6.5, N=3.2, \xi=23$
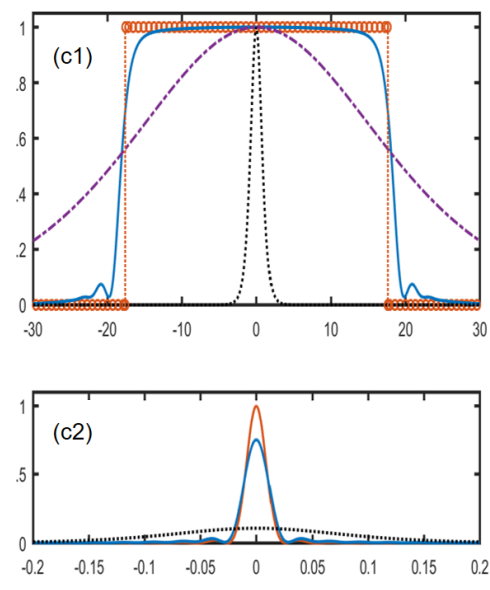

Figure 2. Generation of close-to-Fourier transform limited parabolic [subplot (a)] and rectangular [subplot (c)] waveforms, and highly chirped triangular waveform [subplot (c)] with respective durations of $10 T_{i n}, 20 T_{i n}$, and

$50 T_{i n}$. The temporal and spectral intensity profiles (panels 1 and 2, respectively) generated at the optimum operating points are plotted with blue lines. Also shown are the target temporal profiles (red open circles), the 
temporal and spectral profiles of the input pulses to the system (dotted black curves), and the temporal profiles obtained after the initial dispersive propagation stage (dash-dotted purple curves).

\section{NONLINEAR SCULPURING IN AN ALL-FIBER FIGURE-8 LASER}

Next, we discuss a new design of a model-locked all-fibre Figure-8 laser employing a nonlinear amplifying loop mirror (NALM) with two active fibre segments and two independently controlled pump-power modules (Fig. 3) $[10,11]$. This laser layout combines the reliability and robustness of conventional Figure- 8 lasers with the flexibility of nonlinear-polarisation-evolution lasers, providing access to a variety of generation regimes with a relatively wide adjustment range of the pulse parameters. Moreover, it enables reliable and reproducible live electronic adjustment of the lasing regimes.

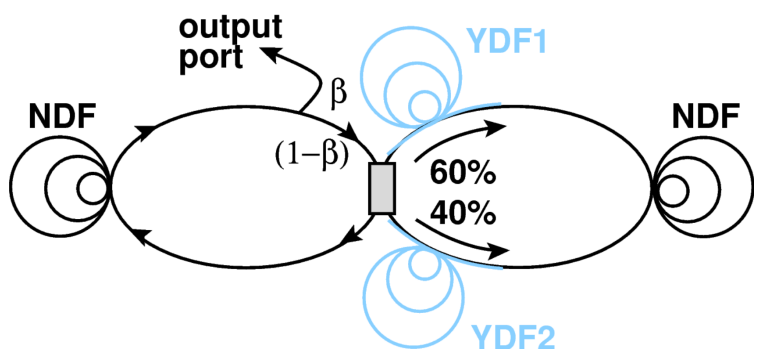

Figure 3. Schematic of the laser. YDF: ytterbium-doped fibre; NDF: normally dispersive fibre.

In this work, we numerically explore the broad range of operating states of the laser that can be accessed through independent control of the pump powers in the two gain segments and the laser output coupling ratio, $\beta$ [12]. We use a piece-wise propagation model for the laser, in which propagation in the fibres follows a standard modified NLSE including gain saturation and spectral response for the active segments. The gain coefficient amplitude is dependent on the average signal and pump powers, the average power dynamics being described by standard rate equations. In a first approach we perform numerical simulations of the propagation model. However, such a study is a costly exercise, the computation of each point in the three-dimensional parameter space of $\beta$, total pump level delivered to the active fibres, $P_{\mathrm{T}}=P_{p, 1}+P_{p, 2}$, and fractional pump power, $R=P_{p, 1} / P_{\mathrm{T}}$, requiring approximately 2 hours on the CPU of a standard personal computer. Therefore, in a second approach we use a NN based model trained on the data from numerical simulations to make predictions across a larger range of the data domain. The algorithm handles almost instantly the whole parameter space.

Figure 4(a) shows various characteristics of the output pulses from the laser as obtained from NLSE numerical simulations for variable $\left(\beta, P_{\mathrm{T}}, R\right)$ parameters. Remarkably, while $P_{\mathrm{T}}$ is varied here by less than $7 \mathrm{~dB}$, the pulse peak power and temporal width display potential variation ranges of nearly $25 \mathrm{~dB}$ and $15 \mathrm{~dB}$, respectively. As a measure of the pulse shape we use here the excess kurtosis, where positive (negative) excess kurtosis equals longer (shorter) and fatter (thinner) tails than a Gaussian shape. As we can see in Fig. 4(a2), low-energy pulses feature positive or slightly negative excess kurtosis, while the excess kurtosis becomes increasingly more negative with increasing pulse energy. The very large range of excess kurtosis values that are observed, from -1.15 to 0.85 , indicates that a broad variety of pulse shapes can be obtained directly at the laser output without the need for external shaping. Indeed, as Fig. 4(b) shows, slight tuning of the pump power ratio at a fixed total pump power enables the generation of pulses with parabolic or flat-top temporal waveforms from the laser cavity.

\section{CONCLUSIONS}

We have presented a general approach to determine the parameters of fibre-based passive nonlinear pulse shaping systems that are required to generate pulses with preset temporal features. Within our approach, the nonlinear shaping process is reduced to a numerical optimisation problem over a three-dimensional space, where the intersections of different surfaces provide the means to quickly identify the sets of parameters of interest. We have also shown that this optimisation problem can be solved efficiently by application of a regression model based on a NN algorithm. Further, we have presented an in-depth numerical characterisation of the various pulse generation regimes of a novel NALM laser with a second segment of active fibre and corresponding pump source, which can be accessed through independent control of the pump powers in the two gain segments and the laser output coupling ratio. We have shown that the application of a NN-based regression model provides a rapid and precise identification of the attainable output pulse properties. A large variety of pulse waveforms can be achieved also in this system. 

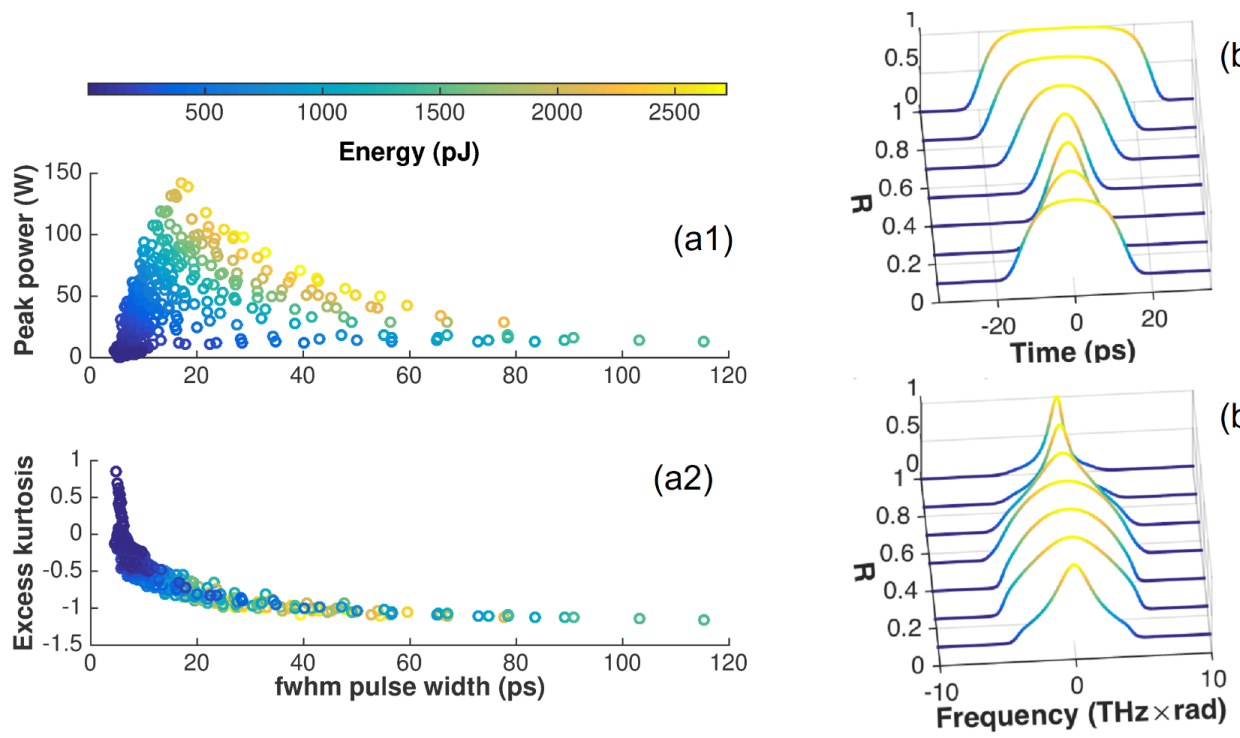

Figure 4. (a) Scatter plots of output pulse characteristics from the laser as obtained from NLSE numerical simulations for variable $\left(\beta, P_{T}, R\right)$ parameters. (a1) Pulse peak power and (a2) excess kurtosis as a function of fwhm pulse width. The points are colour-coded to display the pulse energy. (b) Evolution of the pulse temporal (b1) and spectral (b2) intensity profiles with the fractional pump power $R$ for an output coupling factor of 0.75 and a total pump power level delivered to the active fibres of $11 \mathrm{~W}$.

\section{ACKNOWLEDGEMENTS}

We acknowledge the support of the Institut Universitaire de France (IUF). We also thank K. Hammani for fruitful discussions.

\section{REFERENCES}

[1] F. Zhang, X. Ge, and S. Pan, "Triangular pulse generation using a dual-parallel Mach-Zehnder modulator driven by a single-frequency radio frequency signal," Opt. Lett., vol. 38, no. 21, pp. 4491-4493, 2013.

[2] D. S. Wu, D. J. Richardson, and R. Slavík, "Optical Fourier synthesis of high-repetition-rate pulses," Optica, vol. 2, no. 1, pp. 18-26, 2015.

[3] C. Finot, "40-GHz photonic waveform generator by linear shaping of four spectral sidebands," Opt. Lett., vol. 40, no. 7, pp. 1422-1425, 2015.

[4] S. Boscolo, A. I. Latkin, and S. K. Turitsyn, "Passive nonlinear pulse shaping in normally dispersive fiber systems," IEEE J. Quantum Electron., vol. 44, no. 12, pp. 1196-1203, 2008.

[5] C. Finot, J. M. Dudley, B. Kibler, D. J. Richardson, and G. Millot, "Optical parabolic pulse generation and applications," IEEE J. Quantum Electron., vol. 45, no. 11, pp. 1482-1489, 2009.

[6] C. Finot and S. Boscolo, "Design rules for nonlinear spectral compression in optical fibers," J. Opt. Soc. Am. $B$, vol. 33, no. 4, pp. 760-767, 2016.

[7] A. A. Kutuzyan, T. G. Mansuryan, G. L. Esayan, R. S. Akobyan, and L. K. Mouradian, "Dispersive regime of spectral compression," Quantum Electron., vol. 38, no. 4, pp. 383-387, 2008.

[8] G. P. Agrawal, Nonlinear Fiber Optics, Fourth Edition. San Francisco, CA: Academic Press, 2006.

[9] C. Finot, I. Gukov, K. Hammani, and S. Boscolo, "Nonlinear sculpturing of optical pulses with normally dispersive fiber-based devices," Opt. Fiber Technol., vol. 45, pp. 306-312, 2018.

[10] S. Kobtsev, A. Ivanenko, A. Kokhanovskiy, and S. Smirnov, "Electronic control of different generation regimes in mode-locked all-fibre F8 laser," Laser Phys. Lett., vol. 15, no. 4, p. 045102, 2018.

[11] A. Kokhanovskiy, A. Ivanenko, S. Kobtsev, S. Smirnov, and S. K. Turitsyn, "Machine learning methods for control of fibre lasers with double gain nonlinear loop mirror," Sci. Rep., vol. 9, no. 1, p. 2916, 2019.

[12] S. Boscolo, C. Finot, I. Gukov, and S. K. Turitysn, "Performance analysis of dual-pump nonlinear amplifying loop mirror mode-locked all-fibre laser," 2019, Submitted. 